PROCEEDINGS OF THE

AMERICAN MATHEMATICAL SOCIETY

Volume 130, Number 10, Pages 3005-3015

S 0002-9939(02)06392-X

Article electronically published on March 15, 2002

\title{
POSITIVE EIGENVALUES OF SECOND ORDER BOUNDARY VALUE PROBLEMS AND A THEOREM OF M. G. KREIN
}

\author{
STEVE CLARK AND DON HINTON
}

(Communicated by Carmen Chicone)

\begin{abstract}
Conditions are given which guarantee that the least real eigenvalue is positive for certain boundary value problems for the vector-matrix equation $-y^{\prime \prime}+p(x) y=\gamma w(x) y$. This leads to conditions which guarantee the stable boundedness, according to Krein, for solutions of $y^{\prime \prime}+\lambda p(x) y=0$ with certain real values of $\lambda$. As a consequence, a result first stated by Krein is proven.
\end{abstract}

\section{INTRODUCTION}

We determine conditions under which the lowest real eigenvalue is positive for certain second order boundary value problems. Both scalar and matrix linear differential equations are considered. For the scalar case, problems consist of

$$
-y^{\prime \prime}+p(x) y=\gamma w(x) y, \quad 0 \leqslant x \leqslant T,
$$

where $p, w$ are real-valued and Lebesgue integrable on $[0, T]$, where $w(x)>0$ a.e., and where boundary conditions are either of Dirichlet type,

$$
y(0)=y(T)=0,
$$

or of antiperiodic type,

$$
y(0)+y(T)=y^{\prime}(0)+y^{\prime}(T)=0 .
$$

As is known, these problems are self-adjoint and have a sequence of eigenvalues increasing to infinity. We let $\Lambda_{0}$ denote the lowest eigenvalue for the boundary value problem given by (1.1) and (1.2), and let $\mu_{0}$ denote the lowest eigenvalue for the problem given by (1.1) and (1.3). Note that $\Lambda_{0} \geqslant \mu_{0}$ as can be seen from the Rayleigh quotient expressions for $\Lambda_{0}$ and $\mu_{0}$.

Showing that $\mu_{0}>0$ is important in establishing the stability of solutions for differential equations with periodic coefficients. We make such an application in section 3. More generally, the problem of showing that the first eigenvalue is positive for the boundary value problem given by (1.1) and (1.2) is equivalent to showing that equation (1.1) is disconjugate on $[0, T]$ for both the scalar and vector-matrix cases, i.e., no nontrivial solution has two zeros in $[0, T]$. The problem of disconjugacy is equivalent to the existence of a solution on $[0, T]$ of the associated Riccati equation. Important applications of disconjugacy may be found in many areas, for example, control theory. The problem of disconjugacy, in turn, is related to

Received by the editors September 8, 2000 and, in revised form, May 14, 2001.

2000 Mathematics Subject Classification. Primary 34C10, 34L15; Secondary 34B24, 34D10.

Key words and phrases. Stable boundedness, positive eigenvalues, Opial inequality. 
the broader question of oscillation. For the scalar case this involves determining when solutions of (1.1) have infinitely many zeros on the interval. General results on disconjugacy may be found in the books by Coppel [10], Hartman [14, and Reid [20, 21]. Similar results exist for the discrete analog of (1.1) and may be found in the book by Ahlbrandt and Peterson [2].

The motivation for this work was a missing proof of a result of Krein [15] on stability of solutions of equations with periodic coefficients. The equations considered are those given by

$$
y^{\prime \prime}+\lambda p(x) y=0, \quad \int_{0}^{T} p(x) d x=0,
$$

where the real function $p$ is periodic of period $T$, Lebesgue integrable on $[0, T]$, and nonzero on a subset of $[0, T]$ with positive measure. It is stated in [15, p. 96] that (1.4) is stably bounded, i.e., all solutions of (1.4) are bounded on $(-\infty, \infty)$ with the same being true for all periodic perturbations of $p$ of sufficiently small norm in $\mathcal{L}^{1}[0, T]$, if

$$
\frac{|\lambda| T}{8} \int_{0}^{T}|p(x)| d t<1,
$$

where $\mathcal{L}^{p}[a, b]$ represents the space of Lebesgue $p$-integrable functions on $[a, b]$.

Krein omitted the $|\lambda|$ in (1.5), but it was clearly intended. Krein states that this result was proved previously in [16], and will be proved again in section 8 of [15]. Curiously, the proof appears in neither place. Professors Andreas Hinz and Hubert Kalf have kindly checked the Russian sources and found it missing there also. The proof most likely existed in an earlier version of the manuscript and was left out of the printed version. We give a proof of this result in section 3

In section 2, homogeneous and nonhomogeneous Opial-like inequalities for two functions are given which are analogous to those for one function that may be found in Agarwal and Pang [1], Brown, Hinton and Fink [8], and Mitrinović, Pecarič and Fink [17]. In addition to these inequalities, an index is defined, for functions which are integrable on $[0, T]$, that characterizes an aspect of their oscillatory nature. In section 3, we establish conditions for positivity of the eigenvalues assuming either (1.2) or (1.3) for the scalar case of (1.1) and apply these results to the stable boundedness of (1.4). Cancellations of the positive and negative parts of $p(x)$ play a significant role in the results obtained here. These cancellations are affected through the use of the inequalities given in section 2, In section 4, we extend to the vector-matrix case for (1.1) those results given in section 3. These extensions are given for a real potential matrix, $p$, which need not be symmetric. We then apply these results to give conditions under which stable boundedness holds for (1.4) in the vector-matrix setting.

\section{Preliminaries}

We now collect some needed results, and begin by quoting two lemmas from [9].

Lemma 2.1. If $f$ is a real function Lebesgue integrable on $[a, b]$, then there exist $x_{1}, x_{2}$ in $[a, b]$ such that

$$
\inf _{\mu \in(-\infty, \infty)}\left(\max _{a \leqslant x \leqslant b}\left|\int_{a}^{x} f(s) d s+\mu\right|\right)=\frac{1}{2}\left|\int_{x_{1}}^{x_{2}} f(s) d s\right| .
$$


As the proof of Lemma 2.1 shows, the optimal choice for $\mu$ is $-(M+m) / 2$ where $M=\max _{a \leqslant x \leqslant b} \int_{a}^{x} f(s) d s$, and $m=\min _{a \leqslant x \leqslant b} \int_{a}^{x} f(s) d s$. For this choice of $\mu$, the integral in (2.1) equals $(M-m) / 2$.

Lemma 2.2. If $f_{1}, f_{2}$ are absolutely continuous real functions on $[a, b]$ with $f_{i}(a)=$ $f_{i}(b)=0$, and $f_{i}^{\prime} \in \mathcal{L}^{2}[a, b], i=1,2$, then

$$
\int_{a}^{b}\left(\left|f_{1}^{\prime}(x) f_{2}(x)\right|+\left|f_{1}(x) f_{2}^{\prime}(x)\right|\right) d x \leqslant\left(\frac{b-a}{2}\right)\left[\int_{a}^{b} f_{1}^{\prime}(x)^{2} d x \int_{a}^{b} f_{2}^{\prime}(x)^{2} d x\right]^{1 / 2} .
$$

Furthermore, equality holds only when $f_{1}$ and $f_{2}$ are linear on $\left[a, \frac{a+b}{2}\right]$, and $\left[\frac{a+b}{2}, b\right]$.

Lemma 2.3. If $f_{1}, f_{2}$ are absolutely continuous real functions on $[a, b]$ with $f_{i}(a)+$ $f_{i}(b)=0$, and $f_{i}^{\prime} \in \mathcal{L}^{2}[a, b], i=1,2$, then

$$
\int_{a}^{b}\left|f_{1}^{\prime}(x) f_{2}(x)+f_{1}(x) f_{2}^{\prime}(x)\right| d x \leqslant\left(\frac{b-a}{4}\right) \int_{a}^{b}\left[f_{1}^{\prime}(x)^{2}+f_{2}^{\prime}(x)^{2}\right] d x .
$$

Proof. The case in which $f_{1}=f_{2}$ has been proven in $[8$. Using this special case, we have

$$
\int_{a}^{b}\left|\left(f_{1}(x) \pm f_{2}(x)\right)\left(f_{1}^{\prime}(x) \pm f_{2}^{\prime}(x)\right)\right| d x \leqslant\left(\frac{b-a}{4}\right) \int_{a}^{b}\left[f_{1}^{\prime}(x) \pm f_{2}^{\prime}(x)\right]^{2} d x .
$$

Then, by the identity,

$$
2\left(f_{1} f_{2}^{\prime}+f_{1}^{\prime} f_{2}\right)=\left(f_{1}+f_{2}\right)\left(f_{1}^{\prime}+f_{2}^{\prime}\right)-\left(f_{1}-f_{2}\right)\left(f_{1}^{\prime}-f_{2}^{\prime}\right),
$$

the triangle inequality, and (2.2), we see that

$$
\begin{aligned}
2 \int_{a}^{b}\left|f_{1} f_{2}^{\prime}+f_{1} f_{2}^{\prime}\right| d x & \leqslant \int_{a}^{b}\left|\left(f_{1}+f_{2}\right)\left(f_{1}^{\prime}+f_{2}^{\prime}\right)\right| d x+\int_{a}^{b}\left|\left(f_{1}-f_{2}\right)\left(f_{1}^{\prime}-f_{2}^{\prime}\right)\right| d x \\
& \leqslant \frac{b-a}{4}\left\{\int_{a}^{b}\left(f_{1}^{\prime}+f_{2}^{\prime}\right)^{2} d x+\int_{a}^{b}\left(f_{1}^{\prime}-f_{2}^{\prime}\right)^{2} d x\right\} \\
& =\frac{b-a}{2} \int_{a}^{b}\left(f_{1}^{\prime}\right)^{2}+\left(f_{2}^{\prime}\right)^{2} d x
\end{aligned}
$$

from which the result follows.

Lemma 2.4. If $f_{1}, f_{2}$ are as in Lemma 2.3, then

$$
\begin{aligned}
\int_{a}^{b} & \left(\left|f_{1}^{\prime}(x) f_{2}(x)\right|+\left|f_{1}(x) f_{2}^{\prime}(x)\right|\right) d x \\
& \leqslant \frac{2(b-a)}{\pi}\left[\int_{a}^{b} f_{1}^{\prime}(x)^{2} d x \int_{a}^{b} f_{2}^{\prime}(x)^{2} d x\right]^{1 / 2} .
\end{aligned}
$$

Moreover, $f_{1}(x)=\sin (\pi(x-a) /(b-a)), f_{2}(x)=\cos (\pi(x-a) /(b-a))$ are extremals.

Proof. A computation shows that

$$
f_{1}(x)=\sin (\pi(x-a) /(b-a)), \quad f_{2}(x)=\cos (\pi(x-a) /(b-a))
$$

produces equality in (2.3). Now, it is known that

$$
\int_{a}^{b}(y)^{2} d x \leq \frac{(b-a)^{2}}{\pi^{2}} \int_{a}^{b}\left(y^{\prime}\right)^{2} d x
$$


holds on the class of absolutely continuous functions $y$ satisfying $y(a)+y(b)=0$. Then to obtain the inequality in (2.3), apply the Cauchy-Schwarz inequality and (2.4) to the left side of (2.3).

Remark 2.5. The author's original statement of Lemma 2.4 included the constant $\sqrt{2}(b-a)$ rather than $2(b-a) / \pi$. Richard Brown [7] of the University of Alabama kindly pointed out the current statement and simple proof now given for this lemma.

For our last lemma, we introduce a construction. Let $p$ be a real-valued, Lebesgue integrable function on $[0, T]$, and let

$$
M=\max _{0 \leq x \leq T} \int_{0}^{x} p(s) d s, \quad m=\min _{0 \leq x \leq T} \int_{0}^{x} p(s) d s .
$$

We note that $M=m$ precisely when $p(x)=0$ for almost every $x \in[0, T]$.

Definition 2.6. Let $l$ denote Lebesgue measure. Let $M$ and $m$ be as defined in (2.5). Given a positive integer $k$, we say subsets $I_{i} \subset[0, T], i=1, \ldots, r$, form a partial $k$-decomposition of $[0, T]$ for $p$ if $l\left(I_{i} \cap I_{j}\right)=0$ for $i \neq j$, and $(M-m) / k \leq$ $\left|\int_{I_{i}} p(s) d s\right|$ for $i=1, \ldots, r$. For such a decomposition,

$$
\frac{r(M-m)}{k} \leq \sum_{i=1}^{r}\left|\int_{I_{i}} p(s) d s\right| \leq \int_{0}^{T}|p(s)| d s
$$

The largest such $r$, over all such decompositions of $[0, T]$, will be denoted by $I(k)$ and said to be the $k$ index of $p$. We set $I(k)=\infty$ when $M=m$.

First, we note that $I(k) \geq k$. Trivially true when $M=m$, we assume that $p(x) \neq 0$ on a subset of $[0, T]$ with positive measure. There exist $x_{1}, x_{2} \in[0, T]$ where $m=\int_{0}^{x_{1}} p(s) d s, M=\int_{0}^{x_{2}} p(s) d s$. It is sufficient to take $x_{1}<x_{2}$. Hence, there is a sequence $s_{0}=x_{1}<s_{1}<\cdots<s_{k}=x_{2}$ such that $\int_{s_{i-1}}^{s_{i}} p(s) d s=$ $(M-m) / k$ for $i=1, \ldots, k$; thus, we take $I_{i}=\left[s_{i-1}, s_{i}\right]$.

If $\int_{0}^{T} p(s) d s=0$, then we may show that $I(k) \geq 2 k$ by an argument similar to that just given. To begin, we again assume that $M \neq m$ and let $q(x)=\int_{0}^{t} p(s) d s$. Since $q(T)=q(0)$, we may extend $q(x)$ periodically with period $T$. Let $x_{1}, x_{2}$ be as before, and let $m=q\left(x_{3}\right)$, where $x_{3}=x_{1}+T$. As we decomposed $\left[x_{1}, x_{2}\right]$, so too we may decompose $\left[x_{2}, x_{3}\right]$. The subintervals thus obtained in $\left[x_{2}, x_{3}\right]$ which are not contained in $[0, T]$ correspond to integrals over subintervals in $\left[0, x_{1}\right]$ with this exception: If $T$ is not an endpoint, say $s_{j-1}<T<s_{j}$, then the interval $\left[s_{j-1}, s_{j}\right]$ is replaced by the set $\left[s_{j-1}, T\right] \cup\left[0, s_{j}-T\right]$. As a consequence of these remarks, we have the following result.

Lemma 2.7. Suppose $p \in \mathcal{L}^{1}[0, T]$. Let $M$ and $m$ be as in (2.5) and let $I(k)$ be the $k$ index of $p$. Then

$$
M-m \leq \frac{k}{I(k)} \int_{0}^{T}|p(s)| d s
$$

Moreover, if $\int_{0}^{T} p(s) d s=0$, then $I(k) \geq 2 k$. 


\section{SCAlAR EIgenVAlue PROBlems}

In this section we give criteria for positivity of the lowest eigenvalues, $\Lambda_{0}$ and $\mu_{0}$, for the scalar boundary value problems given by (1.1) together with either (1.2) or (1.3) respectively. The result stated by Krein (cf. (1.5) follows as a consequence of Corollary 3.2 .

Theorem 3.1. Let $p \in \mathcal{L}^{1}[0, T]$, and let $M$ and $m$ be as in (2.5). Then,

(i) $\Lambda_{0}>0$ if

$$
\frac{(M-m) T}{4} \leq 1
$$

(ii) $\mu_{0}>0$ if $\int_{0}^{T} p(s) d s=0$ and (3.1) holds.

Proof. Multiplying (1.1) by $y$ and integrating by parts yields

$$
-\left.y^{\prime}(x) y(x)\right|_{0} ^{T}+\int_{0}^{T}\left(y^{\prime}\right)^{2} d x+\left.q(x) y^{2}(x)\right|_{0} ^{T}-2 \int_{0}^{T} q y y^{\prime} d x=\gamma \int_{0}^{T} w y^{2} d x,
$$

where $q(x)=\int_{0}^{x} p(s) d s+\omega$, and $\omega \in \mathbb{R}$ is an arbitrary constant. Given that $y$ is a solution of (1.1), satisfying either (1.2) or (1.3), then $-\left.y^{\prime}(x) y(x)\right|_{0} ^{T}=\left.q(x) y^{2}(x)\right|_{0} ^{T}=$ 0 . Hence,

$$
\begin{aligned}
\int_{0}^{T}\left(y^{\prime}\right)^{2} d x & =\gamma \int_{0}^{T} w y^{2} d x+2 \int_{0}^{T} q y y^{\prime} d x \\
& \leqslant \gamma \int_{0}^{T} w y^{2} d x+2 \max _{0 \leqslant x \leqslant T}|q(x)| \int_{0}^{T}\left|y y^{\prime}\right| d x .
\end{aligned}
$$

Now by Opial's inequality (see Agarwal and Pang [1] or Mitrinović, Pecarič and Fink [17] for (1.2) and Brown, Fink and Hinton [8] for (1.3)),

$$
\int_{0}^{T}\left|y y^{\prime}\right| d x \leqslant \frac{T}{4} \int_{0}^{T}\left(y^{\prime}\right)^{2} d x
$$

Hence, we have

$$
\int_{0}^{T}\left(y^{\prime}\right)^{2} d x \leqslant \gamma \int_{0}^{T} w y^{2} d x+\frac{(M-m) T}{4} \int_{0}^{T}\left(y^{\prime}\right)^{2} d x
$$

where we have applied Lemma 2.1 to $q$ by choosing $\omega=-(M+m) / 2$.

If $M=m$, then $\Lambda_{0}$ and $\mu_{0}$ are positive by (3.2). If $M>m$, then the inequality in (3.4) is strict for a nontrivial solution $y$ of (1.1). Equality in (3.4) implies equality in (3.3). Equality in (3.3) implies that $y$ is a piecewise linear function with one interior node (cf. [1, 8, 17). However, $y$ is a solution of (1.1) so that $y^{\prime}$ is absolutely continuous. As a result, $y$ is not piecewise linear; thus (3.3), and hence (3.4), is strict. The proof is completed upon dividing (3.4) by $\int_{0}^{T}\left(y^{\prime}\right)^{2} d x$.

By Lemma 2.7 we have the following immediate consequence of Theorem 3.1

Corollary 3.2. Let $p \in \mathcal{L}^{1}[0, T]$. Then,

(i) $\Lambda_{0}>0$ if

$$
\frac{T k}{4 I(k)} \int_{0}^{T}|p(s)| d s \leqslant 1 ;
$$

(ii) $\mu_{0}>0$ if $\int_{0}^{T} p(s) d s=0$ and (3.5) holds. 
Example 3.3. Suppose $p(x)=\alpha \sin (j x)$ on $[0,2 \pi]$, with $j$ a positive integer, and let $k=2$ in Lemma [2.7] A computation then shows that $I(2)=4 j$. Since $\int_{0}^{2 \pi}|p(s)| d s=4|\alpha|$ and $\int_{0}^{2 \pi} p(s) d s=0$, then Corollary 3.2 shows that both $\Lambda_{0}$ and $\mu_{0}$ are positive if $|\alpha| \leqslant j / \pi$.

We now show that Corollary 3.2 yields the result of Krein. First we review some properties of periodic equations (cf. Eastham [12]). For real $p(x) \in \mathcal{L}^{1}[0, T]$, periodic of period $T$, let $\nu_{0} \leqslant \nu_{1} \leqslant \ldots$ be the eigenvalues of (1.1) with periodic boundary conditions $y(0)=y(T), y^{\prime}(0)=y^{\prime}(T)$, and let $\mu_{0} \leqslant \mu_{1} \leqslant \ldots$ be the eigenvalues of (1.1) with antiperiodic boundary conditions (1.3). The stability intervals are then given by $\left[\nu_{0}, \mu_{0}\right],\left[\mu_{1}, \nu_{1}\right],\left[\nu_{2}, \mu_{2}\right]$, etc. If $\gamma$ is in the interior of a stability interval of (1.1), the equation has only bounded solutions on $\mathbb{R}$.

Now when $p(x) \neq 0$ on a subset of $[0, T]$ with positive measure, the condition $\int_{0}^{T} p(s) d s \leqslant 0$ implies $\nu_{0}<0$ (cf. [12, p. 42]). If $\mu_{0}>0$, then the equation $-y^{\prime \prime}+p(x) y=0$ has only bounded solutions on $\mathbb{R}$. Hence part (ii) of Corollary 3.2 shows that $-y^{\prime \prime}+\lambda p(x) y=0$ has only bounded solutions on $\mathbb{R}$ if $\int_{0}^{T} p(s) d s=0$ and (1.5) holds, because $I(k) \geqslant 2 k$. If the inequality (1.5) is strict, then we have stable boundedness since the inequality will remain strict under sufficiently small periodic perturbations in the $\mathcal{L}^{1}[0, T]$ norm. And while the example above shows that improvements over (1.5) are possible with the test given in (3.5) when $I(k)>2 k$, we note that (3.1) is both the stronger and potentially simpler test to apply.

Lastly, we note that in the periodic case of (1.4), a self-adjoint operator $H$ can be defined on functions on $[0, \infty)$ by imposing a boundary condition at 0 . A problem that has received a good deal of work, also for non-periodic $p(x)$ and in higher dimensions, is to determine $e(\lambda)$ which is defined as the infimum of the spectrum of $H$. The above mentioned result of Eastham shows that $e(\lambda)<0$ in the periodic case, but gives no information how $e(\lambda)$ varies as the coupling constant $\lambda$ tends to 0 . In the paper by Gesztesy, Graf and Simon [13], it is proved that under general conditions $e(\lambda)$ satisfies bounds of the form $-a \lambda^{2} \leqslant e(\lambda) \leqslant-b \lambda^{2}$ for $|\lambda|$ sufficiently small where $a, b>0$. The problem of finding bounds on eigenvalues has received enormous attention. To mention a small sampling of the one-dimensional results we cite Ashbaugh and Benguria [3], Bandle [4], Bennewitz and Veling [5], and Brown, Hinton, and Schwabik 6]. For numerical results, we cite Davies [11, Plum [18, and Pryce 19.

\section{Matrix eigenvalue problems}

The matrix analog of (1.1) is

$$
-y^{\prime \prime}+P(x) y=\gamma W(x) y, \quad 0 \leqslant x \leqslant T,
$$

where $y(x)$ is an $n$-dimensional vector, where $P(x)$ and $W(x)$ are $n \times n$ real matrixvalued functions that are Lebesgue integrable on $[0, T]$, and where $W(x)$ is positive definite almost everywhere. Let $P(x)^{t}$ denote the transpose of $P(x)$.

We consider eigenvalue problems for (4.1) with boundary conditions of either Dirichlet-type as in (1.2), or antiperiodic-type as in (1.3). $P(x)$ is not assumed to be symmetric so that the eigenvalue problems may not be self-adjoint. However, $\gamma$ is assumed to be a real eigenvalue (so that without loss of generality, we take $y(x) \in \mathbb{R}^{n \times 1}$ ). Conditions are established which guarantee that $\gamma>0$. We begin by first introducing several $n \times n$ real matrices. 
Let $Q(x)$ and $R(x)$ be real $n \times n$ matrix-valued functions given by

$$
Q(x)=\int_{0}^{x} P(s) d s+\Omega
$$

where $\Omega$ is an $n \times n$ constant matrix, and where

$$
R(x)=Q(x)+Q(x)^{t}=\int_{0}^{x}\left(P(s)+P(s)^{t}\right) d s+\Gamma, \quad \Gamma=\Omega+\Omega^{t} .
$$

Let $\mathcal{B}$ and $\widetilde{\mathcal{B}}$ be $n \times n$ real matrices whose entries are defined by

$$
\mathcal{B}_{i j}=\inf _{\Gamma_{i j} \in \mathbb{R}} \max _{0 \leq x \leq T}\left|R_{i j}(x)\right|, \quad \widetilde{\mathcal{B}}_{i j}=\frac{1}{2} \int_{0}^{T}\left|P_{i j}(s)+P_{j i}(s)\right| d s .
$$

Let $M$ and $m$ be $n \times n$ real matrices whose entries are defined by

$$
M_{i j}=\max _{0 \leq x \leq T} \int_{0}^{x}\left(P_{i j}(s)+P_{j i}(s)\right) d s, \quad m_{i j}=\min _{0 \leq x \leq T} \int_{0}^{x}\left(P_{i j}(s)+P_{j i}(s)\right) d s .
$$

By Lemma 2.1 note that $\mathcal{B}=\frac{1}{2}(M-m)$. Hence by Lemma 2.7 .

$$
\mathcal{B}_{i j}=\frac{M_{i j}-m_{i j}}{2} \leq \frac{k}{2 I(k)_{i j}} \int_{0}^{T}\left|P_{i j}(s)+P_{j i}(s)\right| d s \leq \frac{k}{I(k)_{i j}} \widetilde{\mathcal{B}}_{i j},
$$

We now present a generalization of Theorem 3.1 to the vector-matrix setting.

Theorem 4.1. Let $\beta$ represent the largest eigenvalue of the matrix $\mathcal{B}$ defined in (4.4). Then, for the eigenvalue problem given by (4.1) and (1.2), the lowest real eigenvalue $\Lambda_{0}$ is positive when $T \beta \leqslant 4$.

Proof. Multiply both sides of (4.1) by $y^{t}$ and integrate by parts to obtain

$$
\begin{aligned}
\int_{0}^{T}\left(y^{\prime}\right)^{t} y^{\prime} d x & =\gamma \int_{0}^{T} y^{t} W y d x+\left.y^{t} y^{\prime}\right|_{0} ^{T}-\int_{0}^{T} y^{t} Q^{\prime} y d x \\
& =\gamma \int_{0}^{T} y^{t} W y d x+\left.y^{t} y^{\prime}\right|_{0} ^{T}-\left.y^{t} Q y\right|_{0} ^{T}+\int_{0}^{T}\left(\left(y^{\prime}\right)^{t} Q y+y^{t} Q y^{\prime}\right) d x
\end{aligned}
$$

By (1.2), $\left.y^{t} y^{\prime}\right|_{0} ^{T}=\left.y^{t} Q y\right|_{0} ^{T}=0$, and with $R(x)$ is defined in (4.3), we obtain

$$
\int_{0}^{T}\left(y^{\prime}\right)^{t} y^{\prime} d x=\gamma \int_{0}^{T} y^{t} W y d x+\int_{0}^{T}\left(y^{\prime}\right)^{t} R y d x
$$

Using the symmetry of $R(x)$, (4.6) can be written as

$$
\begin{aligned}
\int_{0}^{T}\left(y^{\prime}\right)^{t} y^{\prime} d x=\gamma \int_{0}^{T} y^{t} W y d x+\sum_{i=1}^{m} \int_{0}^{T} y_{i}^{\prime} y_{i} R_{i i} d x & \\
& +\sum_{i>j} \int_{0}^{T}\left(y_{i}^{\prime} y_{j}+y_{i} y_{j}^{\prime}\right) R_{i j} d x .
\end{aligned}
$$

With $\mathcal{B}$ defined in 4.4 we obtain

$$
\begin{aligned}
\int_{0}^{T}\left(y^{\prime}\right)^{t} y^{\prime} d x \leqslant \gamma \int_{0}^{T} y^{t} W y d x+\sum_{i=1}^{n} \mathcal{B}_{i i} \int_{0}^{T}\left|y_{i}^{\prime} y_{i}\right| d x & \\
& +\sum_{i>j} \mathcal{B}_{i j} \int_{0}^{T}\left|y_{i}^{\prime} y_{j}+y_{i} y_{j}^{\prime}\right| d x .
\end{aligned}
$$


By Lemma 2.2 we obtain

$$
\begin{array}{rl}
\int_{0}^{T}\left(y^{\prime}\right)^{t} y^{\prime} d x \leqslant \gamma \int_{0}^{T} y^{t} W y & d x+\sum_{j=1}^{n} \mathcal{B}_{j j} \frac{T}{4} \int_{0}^{T}\left(y_{j}^{\prime}\right)^{2} d x \\
+ & 2 \sum_{i>j} \frac{T}{4} \mathcal{B}_{i j}\left[\int_{0}^{T}\left(y_{i}^{\prime}\right)^{2} d x \int_{0}^{T}\left(y_{j}^{\prime}\right)^{2} d x\right]^{1 / 2}
\end{array}
$$

hence by the symmetry of $\mathcal{B}$ we obtain

$$
\int_{0}^{T}\left(y^{\prime}\right)^{t} y^{\prime} d x \leqslant \gamma \int_{0}^{T} y^{t} W y d x+\frac{T}{4} \xi^{t} \mathcal{B} \xi
$$

where

$$
\xi_{i}=\left[\int_{0}^{T}\left(y_{i}^{\prime}\right)^{2} d x\right]^{1 / 2}, \quad i=1, \ldots, n .
$$

The symmetry of $\mathcal{B}$ also implies that

$$
\beta=\max \left\{v^{t} \mathcal{B} v \mid\|v\|_{2}=1\right\} .
$$

Hence we note, for $\xi$ in (4.10), that (4.9) yields

$$
\xi^{t} \xi \leqslant \gamma \int_{0}^{T} y^{t} W y d x+\frac{T}{4} \beta \xi^{t} \xi
$$

from which the conclusion of the theorem follows as a consequence. Strict inequality in (4.12) follows by reasoning analogous with the scalar case.

As a consequence of Lemma 2.7 the inequality in (4.5) yields the following:

$$
\mathcal{B}_{i j} \leq \sigma(k) \widetilde{\mathcal{B}}_{i j}, \quad \text { where } \quad \sigma(k)=\max _{1 \leq i, j \leq n} \frac{k}{I(k)_{i j}} .
$$

Moreover, if $\int_{0}^{T} P(s) d s=0$, then $I(k)_{i j} \geq 2 k$ and hence $\sigma(k) \leq 1 / 2$. By the symmetry of $\widetilde{\mathcal{B}}$ defined in (4.4), and the characterization given by (4.11) of its largest eigenvalue $\widetilde{\beta}$ we obtain by (4.9) a result analogous to part (i) of Corollary 3.2 .

Corollary 4.2. Let $\widetilde{\beta}$ represent the maximum for the set of eigenvalues of the matrix $\widetilde{\mathcal{B}}$ defined in (4.4). Then, for the eigenvalue problem given by (4.1) and (1.2), the lowest real eigenvalue $\Lambda_{0}$ is positive when $\sigma(k) \widetilde{\beta} T \leqslant 4$.

Before considering our eigenvalue problem using antiperiodic boundary conditions, we first recall that the row-sum norm for the matrix $\mathcal{B}$ is given by

$$
\|\mathcal{B}\|_{\infty}=\max _{1 \leqslant i \leqslant n} \sum_{j=1}^{n} \mathcal{B}_{i j}=\sup \left\{\|\mathcal{B} v\|_{\infty} \mid\|v\|_{\infty}=1\right\},
$$

where $\|v\|_{\infty}=\max _{1 \leqslant i \leqslant n}\left\{\left|v_{i}\right|\right\}$. We next define the matrices $\mathcal{D}$ and $\widetilde{\mathcal{D}}$ by

$$
\mathcal{D}_{i j}=\left\{\begin{array}{ll}
(1 / 4) \mathcal{B}_{i i}, & \text { if } i=j, \\
(1 / \pi) \mathcal{B}_{i j}, & \text { if } i \neq j,
\end{array} \quad \widetilde{\mathcal{D}}_{i j}= \begin{cases}(1 / 4) \widetilde{\mathcal{B}}_{i i}, & \text { if } i=j, \\
(1 / \pi) \widetilde{\mathcal{B}}_{i j}, & \text { if } i \neq j,\end{cases}\right.
$$

where $\mathcal{B}$ and $\widetilde{\mathcal{B}}$ are defined in (4.4). 
Theorem 4.3. Let $\delta$ represent the largest eigenvalue of the matrix $\mathcal{D}$ defined in (4.14). Then, for the eigenvalue problem given by (4.1) and (1.3), the lowest real eigenvalue $\mu_{0}$ is positive if $\int_{0}^{T} P(x) d x=0$ and either

$$
T\|\mathcal{B}\|_{\infty}<4
$$

or

$$
T \delta<1
$$

Proof. Now $\left.y^{t} y^{\prime}\right|_{0} ^{T}=\left.y^{t} Q y\right|_{0} ^{T}=0$, when (1.3) is satisfied, and $\int_{0}^{T} P(x) d x=0$. Hence, multiplying (4.1) by $y^{t}(x)$ and integrating by parts yields (4.7). At this point, we may use either Lemma 2.3 or Lemma 2.4.

By Lemma 2.3. (4.7) yields

$$
\begin{aligned}
\int_{0}^{T}\left(y^{\prime}\right)^{t} y^{\prime} d x \leqslant \gamma \int_{0}^{T} y^{t} W y d x+\sum_{j=1}^{n} & \mathcal{B}_{j j} \frac{T}{4} \int_{0}^{T}\left(y_{j}^{\prime}\right)^{2} d x \\
& +\sum_{i>j} \mathcal{B}_{i j} \frac{T}{4} \int_{0}^{T}\left(\left(y_{i}^{\prime}\right)^{2}+\left(y_{j}^{\prime}\right)\right)^{2} d x .
\end{aligned}
$$

By the symmetry of $\mathcal{B}, 4.17$ can be written as

$$
\begin{array}{rl}
\int_{0}^{T}\left(y^{\prime}\right)^{t} y^{\prime} d x \leqslant \gamma \int_{0}^{T} y^{t} W y & d x+\sum_{i=j} \mathcal{B}_{i j} \frac{T}{4} \int_{0}^{T}\left(y_{i}^{\prime}\right)^{2} d x \\
& +\sum_{i>j} \mathcal{B}_{i j} \frac{T}{4} \int_{0}^{T}\left(y_{i}^{\prime}\right)^{2} d x+\sum_{i<j} \mathcal{B}_{i j} \frac{T}{4} \int_{0}^{T}\left(y_{i}^{\prime}\right)^{2} d x
\end{array}
$$

hence as

$$
\begin{aligned}
\int_{0}^{T}\left(y^{\prime}\right)^{t} y^{\prime} d x & \leqslant \gamma \int_{0}^{T} y^{t} W y d x+\sum_{i=1}^{n} \sum_{j=1}^{n} \mathcal{B}_{i j} \frac{T}{4} \int_{0}^{T}\left(y_{i}^{\prime}\right)^{2} d x \\
& \leqslant \gamma \int_{0}^{T} y^{t} W y d x+\|\mathcal{B}\|_{\infty} \frac{T}{4} \int_{0}^{T}\left(y^{\prime}\right)^{t} y^{\prime} d x
\end{aligned}
$$

from which (4.15) follows.

By Lemmas 2.2] and 2.4, the inequality in (4.7) yields

$$
\begin{array}{rl}
\int_{0}^{T}\left(y^{\prime}\right)^{t} y^{\prime} d x \leqslant \gamma \int_{0}^{T} y^{t} W y & d x+\sum_{j=1}^{n} \mathcal{B}_{j j} \frac{T}{4} \int_{0}^{T}\left(y_{j}^{\prime}\right)^{2} d x \\
& +2 \sum_{i>j} \frac{T}{\pi} \mathcal{B}_{i j}\left[\int_{0}^{T}\left(y_{i}^{\prime}\right)^{2} d x \int_{0}^{T}\left(y_{j}^{\prime}\right)^{2} d x\right]^{1 / 2} .
\end{array}
$$

Now replacing $T / 4$ with $T$ and $\mathcal{B}_{i j}$ with $\mathcal{D}_{i j}$ in (4.8)-(4.12), we see that (4.18) yields

$$
\xi^{t} \xi \leqslant \gamma \int_{0}^{T} y^{t} W y d x+T \xi^{t} \mathcal{D} \xi
$$


with $\xi$ defined in (4.10). As we obtained (4.12), now we obtain

$$
\xi^{t} \xi \leqslant \gamma \int_{0}^{T} y^{t} W y d x+T \delta \xi^{t} \xi
$$

from which (4.16) follows.

By reasoning analogous to that which gave us Corollary 4.2 we now obtain

Corollary 4.4. Let $\tilde{\delta}$ represent the maximum for the set of eigenvalues of the matrix $\widetilde{\mathcal{D}}$ defined in (4.14). Then, for the eigenvalue problem given by (4.1) and (1.3), the lowest real eigenvalue $\mu_{0}$ is positive when $\int_{0}^{T} P(x) d x=0$ and

$$
\sigma(k) \tilde{\delta} T<1,
$$

where $\sigma(k)$ is defined in 4.13.

We now turn to the issue of stable boundedness for the solutions of the system

$$
y^{\prime \prime}+\lambda P(x) y=0
$$

where $P(T+x)=P(x)=P(x)^{t} \in \mathbb{R}^{n \times n}$. In [15, section 5], Krein proves the following result for such systems.

Theorem 4.5 (Krein). Suppose that $\int_{0}^{T} P(x) d x=0$ and that $P(x) \eta \not \equiv 0$ for any constant vector $\eta \neq 0$. Then, there is at least one negative and one positive eigenvalue for the problem given by (4.20) and (1.3). Moreover, if $\lambda_{1}$ is the smallest positive eigenvalue and if $\lambda_{-1}$ is the largest negative eigenvalue, then for $\lambda \in\left(\lambda_{-1}, \lambda_{1}\right)$, $\lambda \neq 0$, all solutions of (4.20) are stably bounded.

In light of Krein's result, we obtain the following:

Theorem 4.6. If any one of the inequalities (4.15), (4.16) or (4.19) holds, then $[-1,1] \subset\left(\lambda_{-1}, \lambda_{1}\right)$ where $\lambda_{-1}$ is the largest negative eigenvalue and $\lambda_{1}$ is the smallest positive eigenvalue for the problem given by (4.20) and (1.3) when $\int_{0}^{T} P(x) d x=$ 0 , and $P(x) \eta \not \equiv 0$ for any constant vector $\eta \neq 0$.

Proof. In (4.1), replace $P(x)$ by $\lambda P(x)$ and set $\gamma=0$. Then, if $\lambda$ is an eigenvalue of (4.20) and (1.3), $\mu_{0} \leqslant 0$. As a result, the inequalities (4.15), (4.16), and (4.19) fail with $\delta$ replaced by $|\lambda| \delta$ and $\tilde{\delta}$ replaced by $|\lambda| \tilde{\delta}$. Given the hypotheses of this theorem, this implies that $|\lambda|>1$.

\section{ACKNOWLEDGMents}

We would like to thank Fritz Gesztesy, Andreas Hinz, and Hubert Kalf for their helpful discussions regarding the literature, and the referee for several helpful suggestions.

\section{REFERENCES}

[1] R. P. Agarwal and P. Y. H. Pang, Opial Inequalities with Applications in Differential and Difference Equations, Kluwer Academic Publishers, Dordrecht, 1995. MR 96h:34001

[2] C. Ahlbrandt and A. Peterson, Discrete Hamiltonian Systems, Kluwer Academic Publishers, Dordrecht, 1996. MR 98m:39043

[3] M. S. Ashbaugh and R. D. Benguria, Eigenvalue ratios for Sturm-Liouville operators, J. Diff. Eqs. 103 (1993), 205-219. MR 94c:34125

[4] C. Bandle, Extremal problems for eigenvalues of the Sturm-Liouville type, In General Inequalities, 5, Birkhäuser, Basel, 1987, 319-339. MR 90k:34020 
[5] C. Bennewitz and E. J. M. Veling, Optimal bounds for the spectrum of a one-dimensional operator, In General Inequalities, 6, Birkhäuser, Basel, 1992, 257-268. MR 94c:34126

[6] R. C. Brown, D. B. Hinton and S. Schwabik, Applications of a one-dimensional Sobolev inequality to eigenvalue problems, Diff. and Integral Eqs. 9 (1996), 481-498. MR 96k:34180

[7] R. C. Brown, private communication, September, 2000.

[8] R. C. Brown, A. M. Fink, and D. B. Hinton, Some Opial, Lyapunov, and De la Valée Poussin inequalities with nonhomogeneous boundary conditions, J. of Inequal. and Appl. 5 (2000), 11-37. MR 2000m:34073

[9] S. Clark and D. Hinton, A Liapunov inequality for linear Hamiltonian systems, Math. Inequalities and Appl. 1 (1998), 201-209. MR 99c:34056

[10] W. A. Coppel, Disconjugacy, Lecture Notes in Mathematics 220, Springer-Verlag, Berlin, 1971. MR 57:778

[11] E. B. Davies, A hierarchical method for obtaining eigenvalue enclosures, Math. of Computation, 69 (2000), 1435-1455. MR 2001a:34148

[12] M. S. P. Eastham, The Spectral Theory of Periodic Differential Equations, Scottish Academic Press, Edinburgh, 1973.

[13] F. Gesztesy, G. M. Graf and B. Simon, The ground state energy of Schrödinger operators, Commun. Math. Phys. 150 (1992), 375-384. MR 93j:47070

[14] P. Hartman, Ordinary Differential Equations, 2nd ed., Birkhäuser, Boston, 1982. MR 83e: 34002

[15] M. G. Krein, On tests for stable boundedness of solutions of periodic canonical systems, Amer. Math. Soc. Transl. Ser. (2), 120 (1983), 71-110.

[16] M. G. Krein, On certain problems of the maximum and minimum of characteristic values and on the Lyapunov zones of stability, Amer. Math. Soc. Transl. Ser. (2), 1 (1955), 163-187. MR 17:484e

[17] D. S. Mitrinović, J. E. Pecarič, and A. M. Fink, Inequalities Involving Functions and their Integrals and Derivatives, Kluwer, Dordrecht, 1991. MR 93m:26036

[18] M. Plum, Eigenvalue inclusions for second order ordinary differential operators by a numerical homotopy method, Z. Angew. Math. Phys. 41 (1990), 205-226. MR 91d:65116

[19] J. D. Pryce, Numerical Solutions of Sturm-Liouville Problems, Clarendon Press, Oxford, 1993. MR 95h:65056

[20] W. T. Reid, Ordinary Differential Equations, Wiley, New York, 1971. MR 42:7963

[21] W. T. Reid, Sturmian Theory for Ordinary Differential Equations, Springer-Verlag, New York, 1980. MR 82f:34002

Department of Mathematics, University of Missouri-Rolla, Rolla, Missouri 65409

E-mail address: sclark@umr.edu

URL: http://www.umr.edu/ clark

Department of Mathematics, University of Tennessee, Knoxville, Tennessee 37996

E-mail address: hinton@math.utk.edu

URL: http://www.math.utk.edu/ ${ }^{\text {hinton }}$ 